\title{
A 1D Multi-Tube Code for the Shockless Explosion Combustion
}

\author{
Giordana Tornow and Rupert Klein
}

\begin{abstract}
Shockless explosion combustion (SEC) has been suggested by Bobusch et al., CST, 186, 2014, as a new approach towards approximate constant volume combustion for gas turbine applications. The SEC process relies on nearly homogeneous autoignition in a premixed fuel-oxidizer charge and acoustic resonances for cyclic recharge. Operation of a single SEC tube has proven to be rather robust in numerical simulations, provided the flow control assures nearly homogeneous autoignition. Configurations with multiple tubes that fire into a common collector plenum preceding the turbine will be needed, however, to avoid excessive fluctuating thermal and mechanical load on the turbine blades. In such a configuration, the resonating tubes will interact with the volume of the plenum, and proper control of these interactions will be an important part of the engine design process. The present work presents an efficient, rough design tool that simulates the firing of such multi-tube SEC configurations into a torus-shaped turbine plenum. Both the tubes and the plenum are represented by computational quasi-one-dimensional gasdynamics modules implemented in a finite volume code for the reactive Euler equations. Suitable tube-to-plenum coupling conditions based on mass, energy, and plenumaxial momentum conservation represent the gasdynamic interactions of all engine components. First investigations utilising this tool reveal considerable dependence of the SEC-tubes' operating conditions on the tube radius and length, and on the tubes' positioning along the plenum torus. The SEC is especially sensitive to the plenum's radius. Misfiring of one of the tubes does essentially not affect the operation of the others and does not even necessarily lead to a shut-down of the disturbed SEC tube.
\end{abstract}

Keywords Approximate constant volume combustion - Shockless explosion combustion

\footnotetext{
G. Tornow $(\varangle) \cdot$ R. Klein

Department of Mathematics, Geophysical Fluid Dynamics, Freie Universität Berlin, Arnimallee 6, 14195 Berlin, Germany e-mail: gtornow@math.fu-berlin.de

R. Klein

e-mail: rupert.klein@math.fu-berlin.de 


\section{Introduction}

The efficiency gain of gas turbines expected by approximate constant volume combustion (aCVC) compared to today's operation mode, the constant pressure deflagration combustion (CPC), is well-known to the community (see for example the analysis of [8]). Various approaches to realising aCVC have been developed and put into practice to different extent and with diverse success. Each approach has its own challenges and drawbacks: the pulsed detonation combustion (PDC) requires a deflagration-to-detonation transition (DDT) in every cycle, the rotating detonation engine (RDE) must be fuelled within a very short time, and the pulse jet engine (PJE) works only in between the ranges of CPC and aCVC, not fully harvesting the potential of constant volume thermodynamic processes. Since 2012, the shockless explosion combustion (SEC) process is under development, [4]. It relies on acoustic resonance for recharge like the PJE, but aims at homogeneous autoignition to approximate constant volume combustion. Thus it avoids the losses associated with the DDT and turbulent deflagration found in the pulsed detonation and pulsed jet engines.

A stringent interpretation of the term "constant volume combustion" demands all parcels of reacting gas to maintain their initial density throughout the process. This is the situation the SEC combustor aims to approximate. During inflow, fresh hot compressed gas enters the SEC tube with a stratified charge that covers about $1 / 3$ of the tube length. The stratification is tuned to induce approximately homogeneous autoignition. With the characteristic time of heat release of realistic fuels being much shorter than the tube's longitudinal acoustic time scale, chemical energy release will take place at approximately constant density, and the pressure will rise substantially within the charge. The ensuing pressure wave transports the released energy down the tube and into the attached turbine plenum. A wave resonance mechanism akin to that utilised in pulse jet engines supports the recharging process.

The conceptual advantage of the SEC over a pulsed detonation combustor (PDC) is that it features much lower peak pressures, shock-related dissipation, and local kinetic energy. This eases the harvesting of the potential efficiency gain from constant volume combustion. Realising nearly homogeneous autoignition in a highly dynamical flow requires very tight control of the fuelling process, however, so that the development of advanced controlling schemes will be crucial for the success of the concept.

A computationally efficient one-dimensional SEC simulation code has been implemented by Berndt [2], that can be used to test and train fuel injection control schemes . It solves the reactive Euler equations by a finite volume method and models the SEC tubes as long-stretched cylinders with axially varying cross-section and a one-dimensional distribution of the state variables along their axis. The software has been employed to study SEC in single-tube operation, e.g., to develop an efficient reduced chemical model designed to probe particular gasdynamic effects of the SEC process [3], or to investigate the sensitivity of the process with respect to various chemical parameters [7]. 
A full-fledged future gas turbine application will likely feature several SEC tubes firing into an annular intermediate plenum which then connects to the turbine. In a first investigation into such arrangements, multiple copies of the above mentioned quasione-dimensional code are coupled here through suitable transition conditions to simulate various arrangements of three SEC tubes coupled to a torus-shaped plenum. The plenum itself is again represented by the same quasi-one-dimensional code, albeit with periodic boundary conditions to model a closed torus. A mass loss from the plenum, determined from a user-defined sub-routine, mimics the turbine mass flow.

The purging and recharge of the SEC tubes relies on pressure wave resonances just as in the pulse jet combustor. As a consequence, a number of interesting questions regarding proper tuning of the interacting non-stationary gasdynamic processes in the coupled tubes and plenum arise. In particular, we study here the influences of the plenum radius and plenum length, the consequences of different arrangements of the SEC tubes along the plenum axis, and the robustness of the cycles in the tubes when one of the fuel supply pipelines is interrupted shortly.

Section 2 briefly summarises the simulation code implemented to investigate these questions. Numerical tests addressing the questions mentioned above are documented in Sect.3. More possible applications and more complex issues for further code development as well as opportunities for improvement and extension are discussed in Sect. 4.

\section{Implementation}

In the following the code utilised for the simulation experiments in Sect. 3 will be described briefly with focus on the main features for the present usage: the quasi-onedimensionality with possibility of lateral in- and outflow and the simplified chemistry model.

\subsection{One-Dimensional Model and Single-Tube Reference Operation}

The basic code that serves as our starting point has been developed by Berndt [2]. It is optimised to work with good accuracy for realistic thermochemical gas properties and to robustly handle strong shocks including detonation waves. It utilises the HartenLax-van Leer (HLL) numerical flux with Einfeldt's correction (HLLEM) to solve the Euler equations for a multi-species ideal gas flow. The MUSCL-Hancock or WENO reconstructions and Strang splitting for chemistry are employed to achieve second order accuracy. See detailed references in [2, 3]. Boundaries are modelled 
by appropriately assigning ghost cell states as usual in this type of scheme. As the SEC tubes are considered to be cylindrical with small aspect ratio, a quasi-onedimensional approximation is adopted. Smooth axial variation of the tube radius is allowed for via inclusion of a suitable pressure source term (see, e.g. [6]).

To represent an SEC tube, the upstream boundary simulates a pressure valve that opens when the pressure in the first grid cell drops below a given compressor pressure value. When the valve opens, non-reactive compressed "air", possibly preheated to a given reference temperature, purges the tube for about half a millisecond, which we take to be the ignition delay time of the compressed and preheated gas. Subsequently, as long as the valve is still open, fuelled mixture enters the tube. A stratified charge (combustible mixture) is generated by varying the fuel mixture fraction of inflowing gas in time. This variation of the gas composition is tuned to produce a homogeneous autoignition after $0.7 \mathrm{~ms}$ under the conditions of standard cyclic operation of a free SEC tube not attached to a plenum.

In this standard cycle, the fresh charge covers about one third of the SEC tube length which is $0.8 \mathrm{~m}$, with a species from the simplified chemistry mechanism modelling a mixture of dimethyl ether (DME) and air close to stoichiometric conditions (details can be found in [3]).

\subsection{Modelling Lateral Inflow and Outflow}

In previous simulations, e.g., in [2, 3], the downstream boundary condition modelled expansion into open space at atmospheric conditions to represent the test rig setup in related laboratory experiments, or into an infinitely large plenum at elevated pressure. Here we initiate the study of interactions between several SEC tubes and a finite size turbine plenum as depicted in Fig. 1. To this end, the code was first extended to allow for modelling lateral mass flow into (or out of) the modelled tube. These processes are represented as they would be in a multidimensional gasdynamics code

Fig. 1 The SEC

tubes-plenum-configuration showing the slanted tubes with the circular plenum and the distribution of one dimensional cells

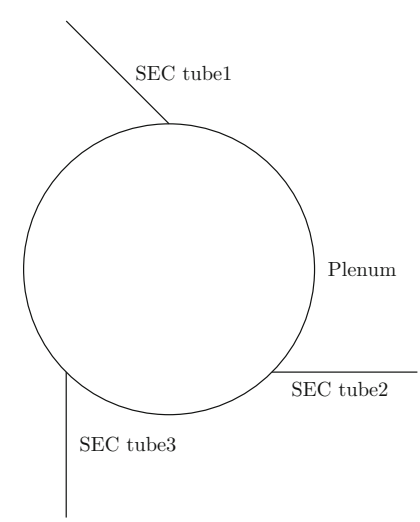




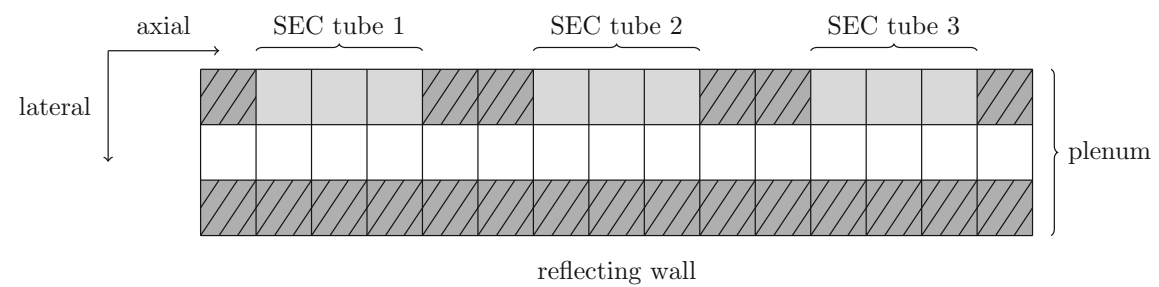

Fig. 2 Modelling the SEC tube-Plenum-Interaction. White cells are the computational domain of the plenum, dark grey cells are solid wall ghost cells and light grey cells are used to couple plenum and SEC tube

using Strang splitting to cover the space dimensions. Thus, for the plenum simulation the code is extended to two space dimensions as indicated in Fig. 2. In the second (lateral) computational direction the computational grid just covers one row of cells representing the main computational domain and two adjacent rows of cells used as dummy cells to impose boundary conditions. The closed tube walls opposite to the exits of the SEC tubes as well as between the SEC tubes on the same side are modelled in the lateral direction by the usual reflecting wall boundary conditions. These guarantee zero mass flux and proper adjustment of the wall pressure. To simulate the exit of the $n$th SEC tube, the flow state found in the last grid cell of the model simulation for that tube at the same time step is imposed in the corresponding dummy cells (light grey cells in the top row of Fig. 2) before processing the gasdynamic step in the lateral direction for the plenum. In turn, the plenum states averaged over the cells corresponding to the width of the attached SEC tube (three cells in the figure) are imposed in the dummy cells of the SEC tube simulations.

In this process, we allow for non-orthogonal intersection of the SEC tubes with the plenum (45 in Fig. 1). By supplying a directional (unit) vector, the user fixes an angle under which the mass flows from the SEC tubes meet the plenum stream. Consistent with the derivation of quasi-onedimensional gasdynamics models, we assume rapid lateral equilibration of all transport processes. This leads to the present model of immediate dissipation of the lateral momentum and kinetic energy upon entry of the burnt gas into the plenum. This is realised by converting the components of momentum in the last cells of the SEC tube simulations in the plenum's lateral direction (vertical direction in the Fig. 2) into internal energy, while the component of momentum aligned with the plenum axis is maintained. In our code this is done by simply setting the momentum in lateral direction to zero but keeping the energy value. This defines the SEC tube states seen by the pertinent plenum's lateral dummy cells (light grey in Fig. 2). All other cells are treated as a reflecting wall as stated above. To account for the feedback of the plenum to one of the SEC tubes, the states in every plenum grid cell that directly couples to this tube are averaged, and this state serves to impose the boundary condition in the ghost cells of the tube. In this fashion, a two-way interaction between the SEC tubes and the plenum is realised. To model 
a possible difference in radius between the SEC tubes and the plenum, the tubes' radii are smoothly increased from their reference radius to that of the plenum. In all simulations shown below, this adaptation of radii covered $2 \%$ of the tube length. This implementation exploits the mentioned possibility of simulating axial variation of radius via a pressure source term in the quasi-one-dimensional computational implementation.

The mass flow out of the plenum that drives the turbine is modelled in the present first approach in a very rudimentary way. The reference single tube SEC run with an opening into infinite space at given mean exit pressure generates a mean mass flux $\dot{m}$ over many cycles. Now, for $n$ tubes attached to the plenum, a total mass of $\Delta t n \dot{m}$ is subtracted from the plenum, equidistributed over all the plenum cells. In doing so, we let the mass deducted from each cell carry the local specific momentum and energy.

The overall algorithm proceeds as follows: Every SEC tube and the plenum are distinct computational domains and treated one after the other beginning with the SEC tubes. Manually, a global time step size is fixed but before every solution step in each domain the stability criteria are tested. The chemical kinetics model (from [3]) and the gasdynamics model based on the Euler equations are then advanced via operator splitting. The computation accounts for the mass flow through the turbine by reducing the density by a user supplied amount in every grid cell scaling with spacial and temporal step width and number of SEC tubes as explained above. For the last operational step, the tubes' interactions with the plenum are determined as also explained above.

\subsection{Reduced Chemical Model for SEC Simulations}

The strongly simplified mechanism for kinetics developed for gasdynamic investigations of the SEC process in [3] is included here. This scheme involves three iconic species: the energy-carrying fuel, an energetically neutral (zero binding enthalpy) "radical" species whose build-up controls the onset of energy release from the fuel component, and a non-reactive product. The model was tuned to mimic the behaviour of a realistic fuel igniting in one stage as far as characteristic time scale ratios of ignition delay and excitation time (heat release rate) are concerned. Reactions are implemented as a sequence of one-step Arrhenius reactions and follow only one path from fuel to radical to product.

\section{Results from Numerical Tests}

In this section we consider a configuration of three SEC tubes coupled to a torusshaped plenum as already seen in Fig. 1 . The SEC tubes are slanted by $45^{\circ}$ relative 

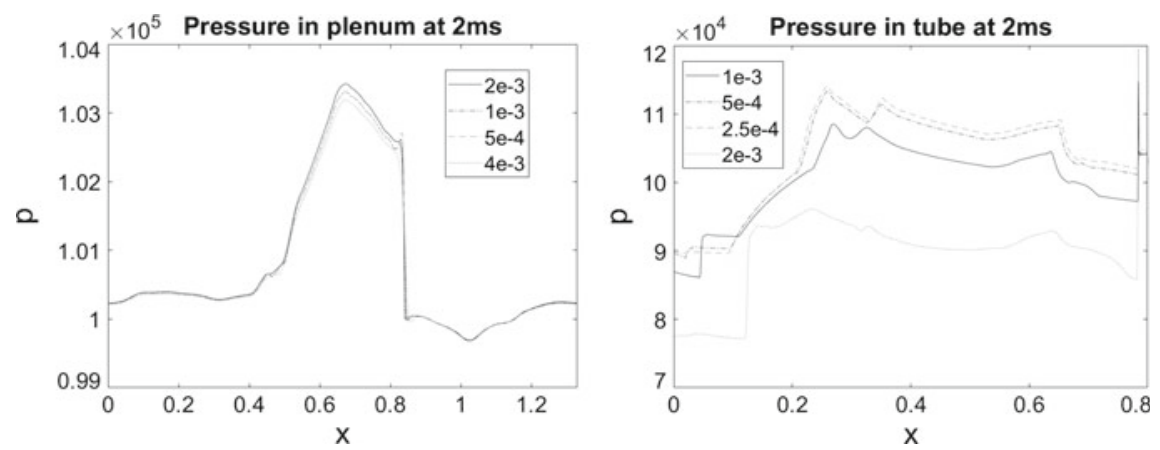

Fig. 3 Pressure in plenum (left) and SEC tube (right) over space, $2 \mathrm{~ms}$ after ignition with three different resolutions: fine (dashed lines), used in further investigations (solid lines) and coarse (dash-dot lines)

to the plenum axis. Their length is $0.8 \mathrm{~m}$ with a resolution of $d x_{T}=8 \times 10^{-4} \mathrm{~m}$ and the basic radius $R_{T}$, except for the radius adjustment towards the plenum, is $0.02 \mathrm{~m}$. The plenum is resolved with a grid cell size of $d x_{P}=1.6 \times 10^{-3} \mathrm{~m}$. To justify the chosen resolution a series of test runs was conducted with only one SEC tube and a plenum of $1.33 \mathrm{~m}$ length with a third, half and double the grid width each. For the plenum resolution Fig. 3 makes clear that even a coarser grid would have been acceptable. As more of the chemistry and dynamics take place in the SEC tubes, these domains are resolved with more grid cells. Figure 3 shows a much larger difference between the coarse and the medium than between the medium and the finer grids, indicating convergence. As the current investigations are designed to qualitatively show the effects of certain parameters on the multi-tube configuration, the medium grid width for the SEC tube seemed to be a good choice. In future works this issue will be tackled by more flexible, non-equidistant meshing.

The fixed time step is chosen to be $d t=5 \times 10^{-5} \mathrm{~ms}$. This value has been extracted from a series of test runs and is found to be a good choice in terms of computational effort.

The right boundary represents the plenum state where the velocity is translated into the SEC tube's coordinate system and other than $x$-direction velocity components are converted to inner energy just as in the opposite case. Because the SEC tube is connected to more than one plenum cell, all plenum cells which interact with the same tube are averaged to form the boundary ghost cell. The plenum is a torus with periodic boundaries.

For all but the tests in Sect.3.1, SEC tubes and plenum simulations are started in the middle of a standard cycle of the given configuration. The initial values for the SEC tubes represent a state in the working cycle after purging and fuelling and just before the next ignition when the radical species is at its highest concentration. The fresh charge occupies about $0.3 \mathrm{~m}$. All tubes are set up equally initially, so they would fire simultaneously. The plenum temperature is initialised everywhere by the 
Table 1 Parameter setting in simulation experiments. $R_{p}$ is the SEC tubes radius, $L_{p}$ the length of the plenum and $x_{T}$ the axial (mid-point) position of the SEC tubes along the plenum

\begin{tabular}{|c|c|c|c|}
\hline \multirow[t]{2}{*}{ Case } & \multicolumn{3}{|l|}{ Param. } \\
\hline & $R_{p}(\mathrm{~m})$ & $L_{p}(\mathrm{~m})$ & $x_{T}(\mathrm{~m})$ \\
\hline \multirow[t]{3}{*}{ Reference } & \multirow[t]{3}{*}{0.08} & \multirow[t]{3}{*}{4} & 1.01 \\
\hline & & & 2.34 \\
\hline & & & 3.68 \\
\hline \multirow[t]{3}{*}{ Sect. 3.1} & \multirow[t]{3}{*}{$0.03,0.06$} & \multirow[t]{3}{*}{4} & 1.01 \\
\hline & & & 2.34 \\
\hline & & & 3.68 \\
\hline \multirow[t]{3}{*}{ Sect. 3.2} & \multirow[t]{3}{*}{0.08} & \multirow[t]{3}{*}{0.5} & 1.01 \\
\hline & & & 2.34 \\
\hline & & & 3.68 \\
\hline \multirow[t]{3}{*}{ Sects. 3.3} & \multirow[t]{3}{*}{0.08} & \multirow[t]{3}{*}{4} & 1.93 \\
\hline & & & 2 \\
\hline & & & 2.07 \\
\hline
\end{tabular}

temperature of the rightmost cell of a SEC tube averaged over four standard cycles. The pressure is chosen to be elevated to $1.1 \mathrm{bar}$.

In the following simulation experiments three variables have been tested for their influence on the SEC cycle: the radius and length of the plenum, and the positioning of the tubes along the plenum. The fourth simulation is a test case with interrupted fuel supply in one of the SEC tubes, which tests the robustness of operation of the tubes. In Table 1 the values of the tested parameters are listed. The configurations with given parameters are compared to the reference case in the respective sections.

\subsection{Plenum Radius}

The refilling of the SEC tube is realised via a suction wave. This is the reflection of the pressure wave from ignition at the downstream end of the SEC tube. Therefore, the radius of the plenum is expected to be crucial for the cyclic operation. We surmise that if it does not behave sufficiently similarly to an ideally open end the refilling will fail. This could be substantiated by the following simulation experiments: The initial values were selected such that the plenum is filled with compressor "air" at rest at $1 \mathrm{bar}$ and $1000 \mathrm{~K}$. The tube is fuelled within the first $0.32 \mathrm{~m}$ with radicals so that ignition is just about to begin. The $0.48 \mathrm{~m}$ downstream are also filled with compressor "air". The plenum radius was set to be 1.5, 3 and 4 times the SEC tube's radius, i.e., within 20 grid cells the SEC tube widens to $0.03,0.06$ and $0.08 \mathrm{~m}$. Figure 4 shows that the cyclic recharge and ignition process fails for the two smaller radii. With larger radius the SEC cycle survives somewhat longer (middle panel), but even from 

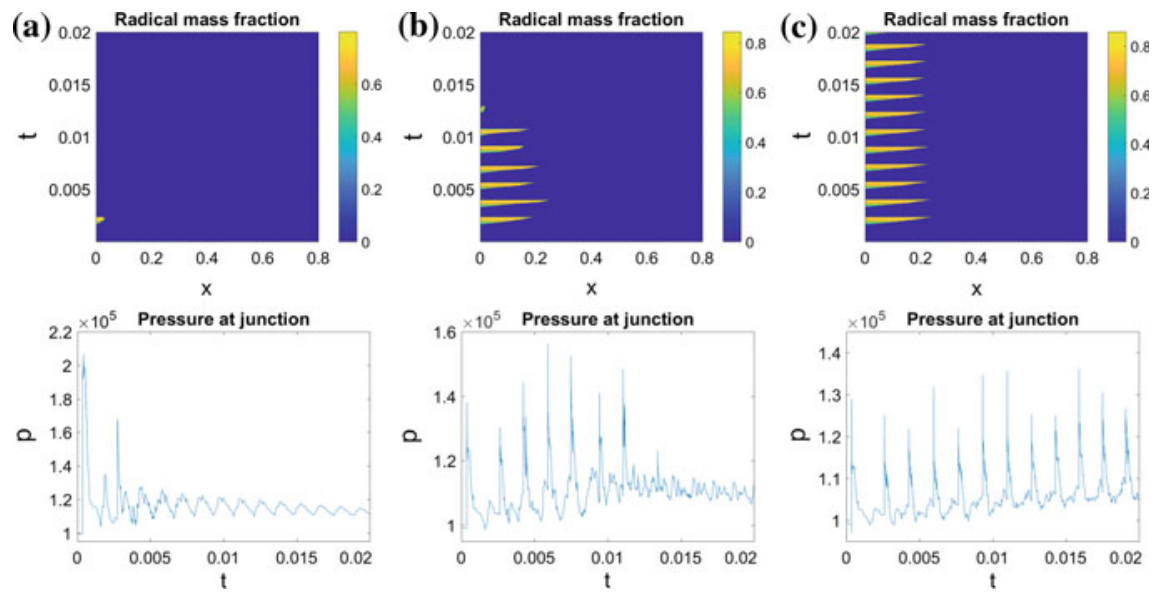

Fig. 4 Influence of plenum radius $R_{P}$ on the SEC cycle. Radical mass fractions and pressure in Pa at SEC tube-plenum junction are shown versus space and time or only time, respectively, for $R_{P}$ $=0.03 \mathrm{~m}=1.5 R_{T}(\mathbf{a}), R_{P}=0.06 \mathrm{~m}=3 R_{T}(\mathbf{b})$, and $R_{P}=0.08 \mathrm{~m}=4 R_{T}(\mathbf{c})$, where $R_{T}$ is the SEC tube radius

the first recharge the stable (right) and the unstable configurations (middle and left) show markedly different behaviour. The stable cycle takes in a larger total load of fresh gas, and the cycles are repeating robustly. We conclude that a plenum radius of $0.08 \mathrm{~m}$ (4 times the SEC tube radius) is sufficient to stabilise the SEC process. This will be the configuration used throughout the following simulations.

\subsection{Length of Plenum}

Here we study the influence of the plenum length, fixing the plenum-to-SEC tube radius ratio to 4 . Results from two simulations are represented in Fig. 5 corresponding to plenum lengths of $0.5 \mathrm{~m}$ and $4 \mathrm{~m}$, respectively. The SEC tubes are operating nearly independently of this parameter. The most interesting change can be seen in the pressure field of the plenum. Especially when comparing the pressure over space at a fixed point in time in the second row of Fig. 5 one can see the smooth structure in the shorter plenum. A clear wave with three maxima developed. This is due to two effects: The most obvious reason is that the ratio of SEC tube radius to plenum length is smaller with smaller plenum and thus the combustion in the tubes raise the pressure in a broader space interval compared to the plenum length. The more interesting reason is that the configuration shown is close to resonance of plenum and SEC tubes. Therefore, a pressure peak from a combustion in the SEC tube hits the traveling pressure wave in the plenum around its maximum.

A future study will aim at investigating the effects of resonance on the SEC process and how they could be exploited. In the following simulation experiments we fix the 

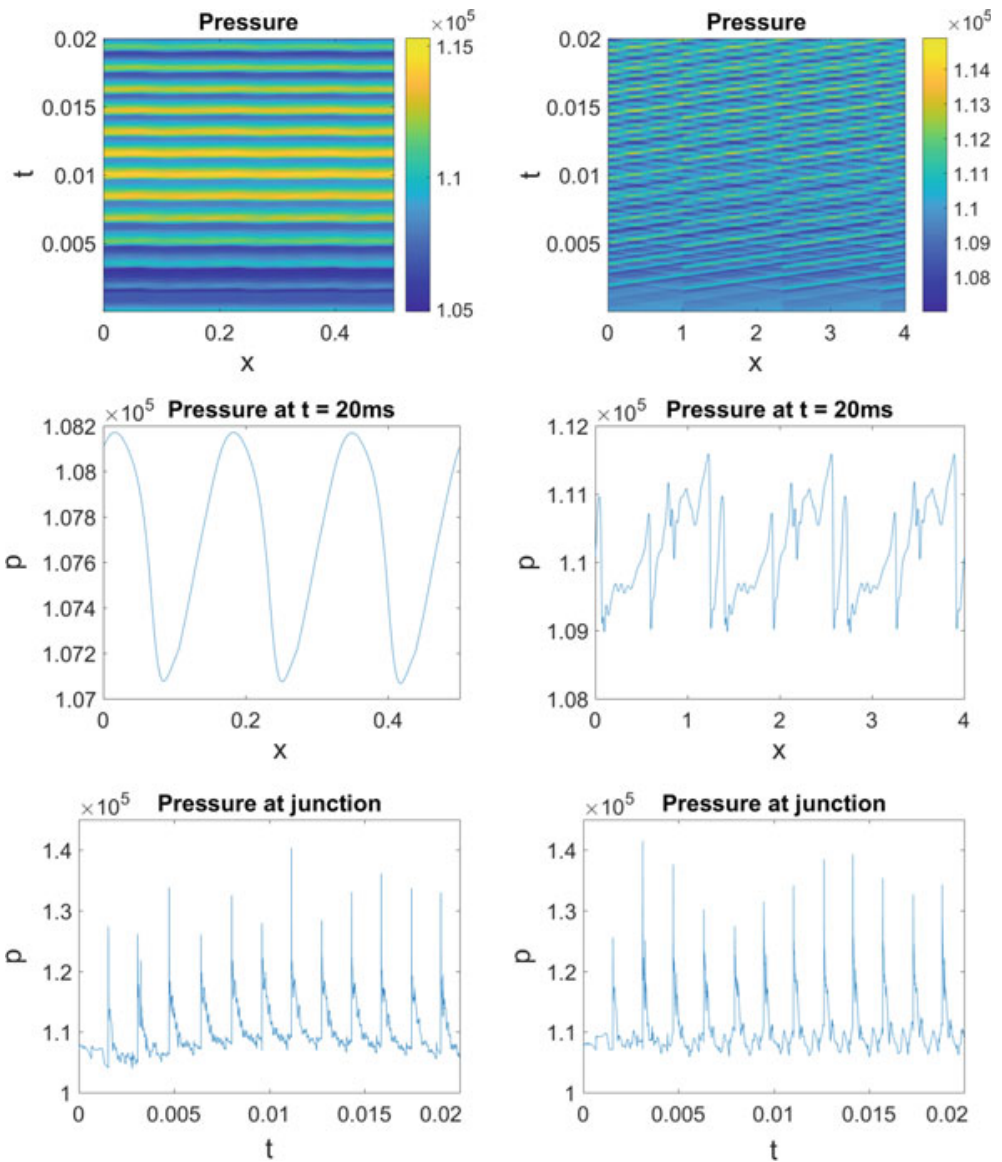

Fig. 5 Influence of plenum length $(0.5 \mathrm{~m}$ left and $4 \mathrm{~m}$ right $)$ on the plenum's pressure field. The figures show the pressure in $\mathrm{Pa}$ in the plenum over space and time (first row), at the simulations last time point $\mathrm{t}=20 \mathrm{~ms}$ over space (second row) and at the junction between SEC tube 1 and the plenum over time (third row)

plenum length to $4 \mathrm{~m}$. For a reference case the very special resonance is not desired as it could shadow the effects of the parameter we wish to study.

\subsection{Positioning of SEC Tubes Along the Plenum}

For the arrangement of SEC tubes along an annular plenum, an equidistant distribution might seem most natural at a first glance. Nonetheless, especially with the results of Sect. 3.2 in mind, we might expect an asymmetric arrangement to enforce the development of clearer and smoother pressure waves. This supposition seems 

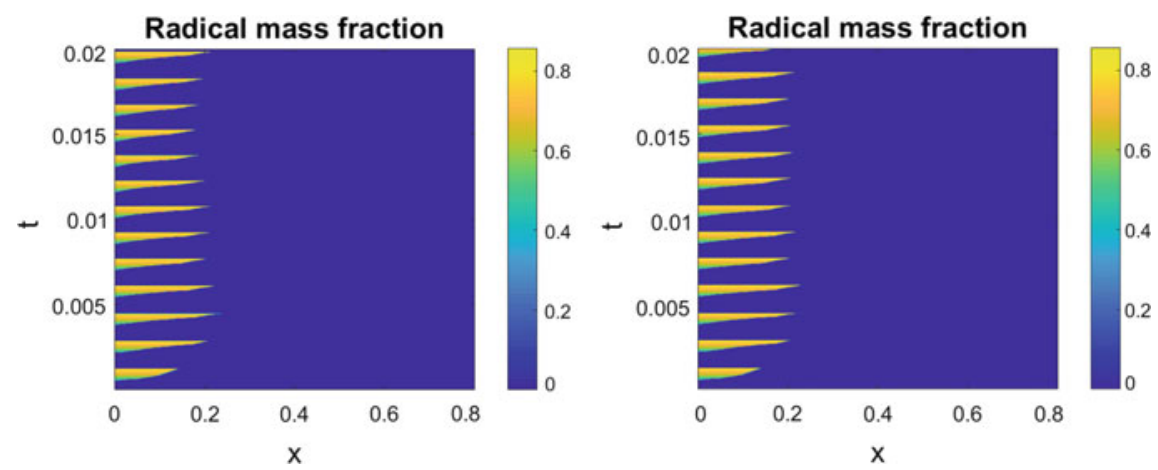

Fig. 6 Influence of SEC tube positioning along the plenum on operation cycle. Left: tube 3 in the bundled case (the one with the highest firing frequency); right: tube 1 in the symmetric case (cycles in the other tubes are equivalent)

to be true as the simulation experiments shown in Figs. 6 and 7 reveal though of course the results differ qualitatively. The current tests represent the extreme cases of equidistantly arranged and very closely bundled SEC tubes with a distance of $0.072 \mathrm{~m}$ ( $\sim 2 \%$ of the plenum length) between neighbouring tubes. Figure 6 displays the fuelling cycles mirrored by the radical mass fractions until time $t=20 \mathrm{~ms}$ in the third bundled SEC tube which is the one with the biggest difference to the reference case and in one of the tubes in the symmetric reference case- the others are equal. Surprisingly, the asymmetric SEC tubes have a slightly higher firing frequency although there are small differences between the bundled tubes. On a longer time scale and with more combustion chambers this could have an important effect on the SEC's efficiency.

The equidistant positioning leads to a pressure field in the plenum (right panel of Fig. 7) with rather fine structures which will result in more homogeneous distributions when turbulent transport is accounted for. In the bundled case we find higher amplitudes intensifying over time and more coarse-grained patterns (left panels of Fig. 7). These could be useful for restarting a shut down SEC tube utilising suction waves in the plenum passing the tube's exit. In-depth investigations of different tube arrangements are to follow in a future study. Nonetheless, within the given time range both configurations work robustly.

\subsection{Interrupted Fuel Pipeline}

The preceding tests were conducted to find a robust configuration to run the SEC as smoothly as possible. In this last investigation we test this robustness. For the time interval of the fourth cycle (5.376-6.99 ms) only compressed "air" without fuel charge is made available for SEC tube 1 . The second and third tube keep operating 

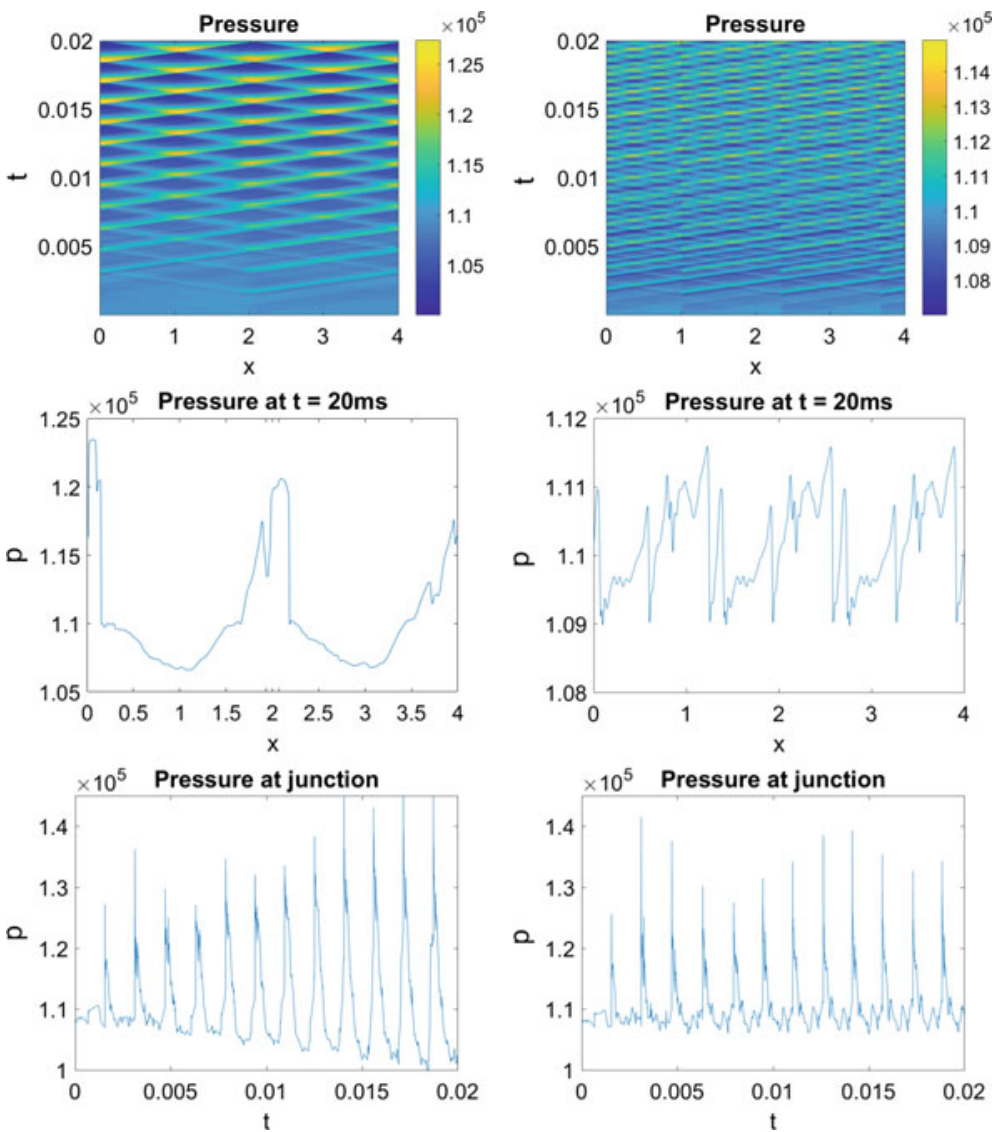

Fig. 7 Influence of SEC tube positioning pressure in the plenum. left: bundled tubes; right: equidistant tubes. The figures show the pressure in $\mathrm{Pa}$ in the plenum over space and time (first row), at the simulations last time point $\mathrm{t}=20 \mathrm{~ms}$ over space (second row) and at the junction between SEC tube 1 and the plenum over time (third row). Please note the different scales of amplitude in the different cases. Equal scales would not reveal patterns in equidistant setting

with minimal disturbances. Hardly visible differences do occur, recognisable when comparing these tube to each other and the undisturbed reference case. This is a consequence of the change in structure in the plenum's pressure field. Unexpectedly, even tube 1 restarts the combustion after the interruption. The cyclic burning is reestablished though unstable. Until now it is unclear whether the combustion will stabilise again over time or die off. This will be the subject of further examinations in the future. Nonetheless, another point can be made for this investigation. In Fig. 9 results from the same test with a slightly different interruption time interval (5.36$6.92 \mathrm{~ms}$ ) are shown. Here tube 1 restarts its cyclic combustion in a stable way. So obviously, there is a tolerance for interruption of fuel supply of about $1.5 \mathrm{~ms}$ probably also depending on the onset of the disturbance. One aim for the future will be to 

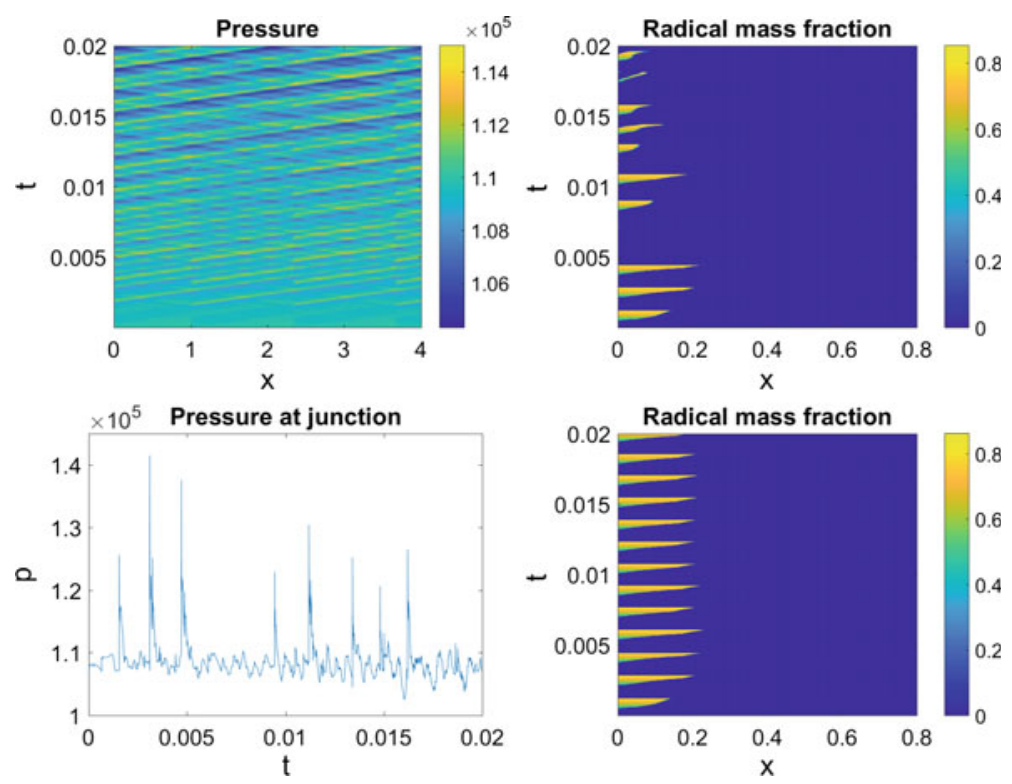

Fig. 8 Interruption of fuel pipeline in tube 1 for time interval 5.376-6.99 ms. The upper figures show the plenum pressure in $\mathrm{Pa}$ (left) and the radical mass fraction in tube 1(right). The lower figures display the pressure in Pa at tube 1's junction to the plenum (left) and the radical mass fraction tube 2 (right)

discover the parameter influencing this tolerance interval to find even more robust configurations and to learn how the restart of a failed tube can be positively influenced by suitable controls.

\section{Conclusion and Outlook}

The previous section has demonstrated the value of the possibility to simulate the highly complex processes going on in SEC tubes coupled to a turbine plenum. We have found interesting hints about what might affect the efficiency and robustness of working cycles and to what extent. Surely, the volume of the plenum must be chosen carefully as we have seen in Sects. 3.1 and 3.2. For the construction of a SEC gas turbine a lower boundary should be found for the studied variables. Although we still need to find a good way to reliably restart a tube after misfiring we can hope for the disturbed tube to reestablish operation and be positive about the others which will keep working nonetheless.

Everything that directly influences the pressure field of the plenum can affect the SEC as can be concluded from the simulation experiments. But not only the plenum configuration and arrangement of the SEC tubes are essential. There are 

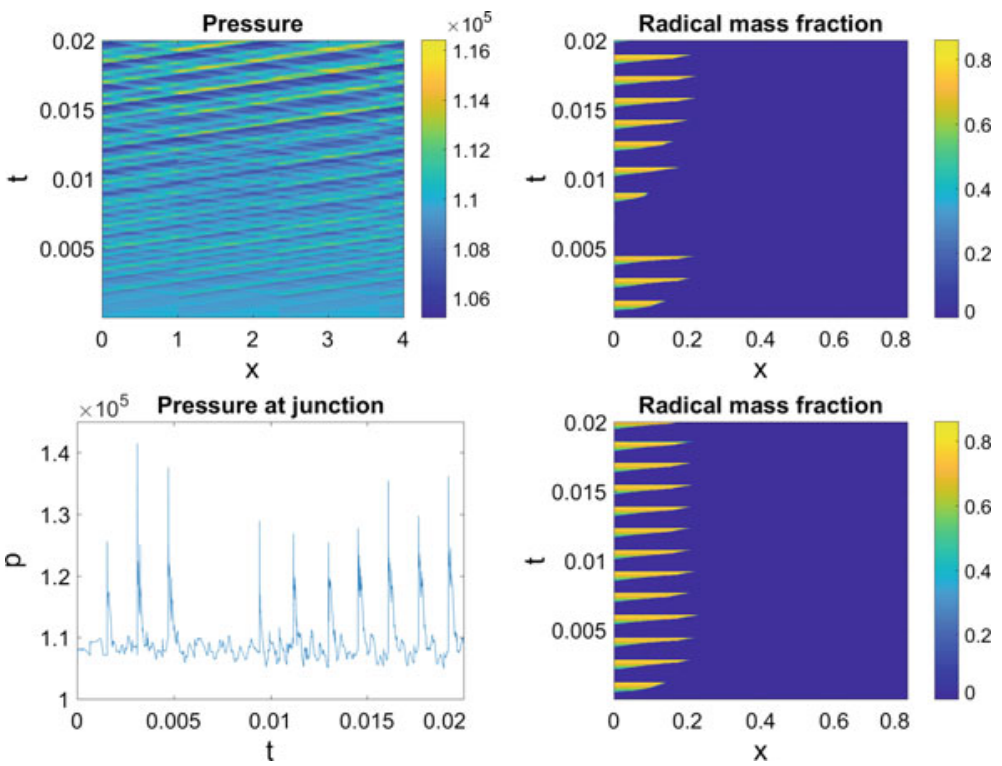

Fig. 9 Interruption of fuel pipeline in tube 1 for 5.36-6.92 ms. The upper figures show the plenum pressure in $\mathrm{Pa}$ (left) and the radical mass fraction in tube 1(right). The lower figures display the pressure in $\mathrm{Pa}$ at tube 1's junction to the plenum (left) and the radical mass fraction tube 2 (right)

more variables to study such as the firing sequence of the tubes, the amount of fuel burnt in every cycle and of course the fuel itself. Other interesting aspects have not yet been investigated but will be the object of future code development and research, such as more realistic representations of the turbine's characteristics, chemical kinetics, molecular and turbulent transport. Nonetheless, as it is today, the coupled quasione-dimensional simulation code can already be used by control engineers for the development and testing of controlling algorithms, and it provides important hints for the design of experimental test rigs in real-world experiments.

Acknowledgements The authors gratefully acknowledge support by Deutsche Forschungsgemeinschaft (DFG) as part of the Collaborative Research Center CRC 1029 "Substantial efficiency increase in gas turbines through direct use of coupled unsteady combustion and flow dynamics", project A03.

\section{References}

1. Anand, V. et al.: Dependence of Pressure, Combustion and Frequency Characteristics on Valved Pulsejet Combustor Geometries, Flow Turbulence and Combustion (2017)

2. Berndt, P.: Mathematical modeling of the shockless explosion combustion, $\mathrm{PhD}$ thesis, Freie Universität Berlin (2016)

3. Berndt, P., Klein, R.: Modeling the kinetics of the shockless explosion combustion. Combust. Flame 175, 16-26 (2017) 
4. Bobusch, B.C., Berndt, P., Paschereit, C.O., Klein, R.: Shockless explosion combustion: An innovative way of efficient constant volume combustion in gas turbines. Combust. Sci. Technol. 186(10-11), 1680-1689 (2014)

5. Lee, J.H., Knystautas, R., Yoshikawa, N.: Photochemical initiation of gaseous detonations. Acta Astronaut. 5(11-12), 971-982 (1978)

6. Shapiro, A.H.: The Dynamics and Thermodynamics of Compressible Fluid Flow. Ronald Press, vol. 1, pp. 1953-54. New York (1953)

7. Zander, L., Tornow, G., Klein, R., Djordjevic, N.: Knock control in shockless explosion combustion by extension of excitation time. AFCC 2018 (same volume) submitted

8. Heiser, W., Pratt, D.T.: Thermodynamic cycle analysis of pulse detonation engines. J. Propuls. Power 18, 68-76 (2002) 